$11-1-2011$

\title{
Modeling Repairable System Failures with Interval Failure Data and Time Dependent Covariate
}

Jayanthi Arasan

University Putra Malaysia, jayanthi@science.upm.edu

Samira Ehsani

UniversityPutra Malaysia, ehsani_samira@yahoo.com

Follow this and additional works at: http://digitalcommons.wayne.edu/jmasm

Part of the Applied Statistics Commons, Social and Behavioral Sciences Commons, and the Statistical Theory Commons

\section{Recommended Citation}

Arasan, Jayanthi and Ehsani, Samira (2011) "Modeling Repairable System Failures with Interval Failure Data and Time Dependent Covariate," Journal of Modern Applied Statistical Methods: Vol. 10 : Iss. 2 , Article 19.

DOI: $10.22237 /$ jmasm/1320121080

Available at: http://digitalcommons.wayne.edu/jmasm/vol10/iss2/19 


\title{
Modeling Repairable System Failures with Interval Failure Data and Time Dependent Covariate
}

\author{
Jayanthi Arasan Samira Ehsani \\ University Putra Malaysia, \\ Malaysia
}

An application of a repairable system model for interval failure data with a time dependent covariate is examined. The performance of several models based on the NHPP when applied to real data on ball bearing failures is also explored. The best model for the data was selected based on results of the likelihood ratio test. The bootstrapping technique was applied to obtain the variance estimate for the estimated expected number of failures. Results demonstrate that the proposed model works well and is easy to implement, in addition the bootstrap variance estimate provides a simple substitute for the traditional estimate.

Key words: Interval, repairable, NHPP, covariate, bootstrap.

\section{Introduction}

A repairable system is a system that can be restored back to functionality after a failure has occurred. The period where the system is unable to function is referred to as repair time and is assumed to be negligible. Grouped data, also known as interval failure data occurs when a component's failure time falls within a certain interval $\left(t_{i-i}, t_{i}\right)$ where $t_{i-i}$ is the lower inspection time and $t_{i}$ is the upper inspection time in the $i^{t h}$ interval. In reliability this phenomenon occurs when components are inspected periodically to carry out maintenance or repair actions. These types of data often arise in the medical field where patients are examined periodically, for example every 3 or 6 months, so the exact failure time is typically unknown.

Many stochastic models have been developed to describe the failure rate of a nonhomogenous Poisson process (NHPP) such

Jayanthi Arasan is a Senior Lecturer in the Department of Mathematics. Email: jayanthi@science.upm.edu.my. Samira Ehsani is a post graduate student in the Department of Mathematics.

Email: ehsani_samira@yahoo.com. as the power law model proposed by Crow (1974) and based on the ideas of Duanne (1964). Other popular models are the log linear proposed by Cox and Lewis (1966) and linear models discussed by Vesely (1977) and Atwood (1992). Lawless and Thiagarajah (1996) introduced an important repairable system model that incorporates both time trends and renewal behavior, known as a proportional intensity model. Guo, et al. (2006) proposed a proportional intensity model that is based on the powerlaw model. Guo, et al. (2007) also developed a new general repair model based on the expected cumulative number of failures to capture the repair history. Samira and Arasan (2009) extended the model to include a time dependent covariate and applied it to pipe failures in water networks.

Other literature on repairable system models and recurrent events includes Brown (1975), Gasmi, et al. (2003), Kaminskiy and Krivtsov (1998), Kijima and Sumita (1986), Kijima (1989), Wang and Pham (1996) and Yanez, et al. (2002). Park, et al. (2008) presented an application of the log-linear and power law models for interval failure data in water distribution systems.

More details regarding recurrent event models for grouped and interval failure data can also be found in Meeker and Escobar (1998), 


\section{ARASAN \& EHSANI}

Lawless and Zhan (1998) and Cook and Lawless (2007).

\section{The Model}

Most recurrent event data, such as in the case of repairable systems, usually has recurrence times that are not be independent. The most widely used models for recurrence data are those based on the non-homogenous Poisson process, mainly the power law and loglinear models. This research extends the power law model to incorporate the analysis of grouped or interval failure data while accommodating the effect of covariates or other factors that may affect or contribute to system failure. Thus, the failure intensity or recurrence rate can be described as $\nu(t)=a b t^{b-1} e^{g x(t)}$, where $x(t)$ is a time dependent covariate that may impact system failure.

Thus, the proposed model takes into account both the effect of time and a time dependent covariate on the recurrence rate of a system. Because it is dealing with interval failure data - and there can be more than one failure in any time interval - the number of intervals is always less or equal to number of failures observed.

Suppose $d_{i}$ is the number of failures in the $i^{t h}$ interval and $x\left(t_{i}\right)$ is the value of covariate at time $t_{i}$. The expected number of recurrences

$$
\mu\left(t_{i-1}, t_{i}\right)=E\left[N\left(t_{i-1}, t_{i}\right)\right]=\int_{t_{i-1}}^{t_{i}} \nu(u) d u,
$$

where $i=1,2, \cdots, n$.

If the intervals are contiguous, the Poisson process log-likelihood for a series of $n$ time intervals is:

$$
\begin{aligned}
L(a, b, g)= & \sum_{i=1}^{n} d_{i} g x\left(t_{i}\right) \ln \left[a\left(t_{i}^{b}-t_{i-1}^{b}\right)\right]- \\
& {\left[a \mathrm{e}^{g x\left(t_{i}\right)}\left(t_{i}^{b}-t_{i-1}^{b}\right)\right]-\ln \left(d_{i} !\right) . }
\end{aligned}
$$

The first and second derivatives of the loglikelihood function are as follows:

$$
\begin{aligned}
\frac{\partial L(a, b, g)}{\partial a}= & \sum_{i=1}^{n} \frac{d_{i}}{a}-\exp \left(g x\left(t_{i}\right)\right)\left(t_{i}^{b}-t_{i-1}^{b}\right), \\
\frac{\partial L(a, b, g)}{\partial b}= & \sum_{i=1}^{n} \frac{d_{i}\left(t_{i}^{b} \ln \left(t_{i}\right)-t_{i-1}^{b} \ln \left(t_{i-1}\right)\right)}{\left(t_{i}^{b}-t_{i-1}^{b}\right)}- \\
& \exp \left(g x\left(t_{i}\right)\right) a\left(t_{i}^{b} \ln \left(t_{i}\right)-t_{i-1}^{b} \ln \left(t_{i-1}\right)\right), \\
\frac{\partial L(a, b, g)}{\partial g}= & \sum_{i=1}^{n} d_{i} x\left(t_{i}\right)-x\left(t_{i}\right) \exp \left(g x\left(t_{i}\right)\right) \\
& a\left(t_{i}^{b}-t_{i-1}^{b}\right), \\
\frac{\partial^{2} L(a, b, g)}{\partial a^{2}}= & \sum_{i=1}^{n}-\frac{d_{i}}{a^{2}}, \\
\frac{\partial^{2} L(a, b, g)}{\partial a \partial b}= & \sum_{i=1}^{n}-\exp \left(g x\left(t_{i}\right)\right)\left(t_{i}^{b} \ln \left(t_{i}\right)-t_{i-1}^{b} \ln \left(t_{i-1}\right)\right), \\
\frac{\partial^{2} L(a, b, g)}{\partial a \partial g}= & \sum_{i=1}^{n}-x\left(t_{i}\right) \exp \left(g x\left(t_{i}\right)\right)\left(t_{i}^{b}-t_{i-1}^{b}\right), \\
\frac{\partial^{2} L(a, b, g)}{\partial b^{2}}= & \sum_{i=1}^{n} \frac{d_{i}\left(t_{i}^{b} \ln \left(t_{i}\right)^{2}-t_{i-1}^{b} \ln \left(t_{i-1}\right)^{2}\right)}{\left(t_{i}^{b}-t_{i-1}^{b}\right)}- \\
& -\frac{d_{i}\left(t_{i}^{b} \ln \left(t_{i}\right)-t_{i-1}^{b} \ln \left(t_{i-1}\right)\right)^{2}}{\left(t_{i}^{b}-t_{i-1}^{b}\right)^{2}} \\
& -\exp \left(g x\left(t_{i}\right)\right) a\left(t_{i}^{b} \ln \left(t_{i}\right)^{2}-t_{i-1}^{b} \ln \left(t_{i-1}\right)^{2}\right), \\
\frac{\partial^{2} L(a, b, g)}{\partial g^{2}}= & \sum_{i=1}^{n}-x\left(t_{i}\right)^{2} \exp \left(g x\left(t_{i}\right)\right) a\left(t_{i}^{b}-t_{i-1}^{b}\right) . \\
\frac{\partial^{2} L(a, b, g)}{\partial b \partial g}= & \sum_{i=1}^{n}-x\left(t_{i}\right) \exp \left(g x\left(t_{i}\right)\right) a\left(t_{i}^{b} \ln \left(t_{i}\right)\right),
\end{aligned}
$$

The extended power law model allows interval failure data to be analyzed by incorporating the effect of time and covariates simultaneously. Occasionally, the effect of covariates are insignificant, thus, the reduced form of the model may prove to be a better fit for the data; this can be obtained by setting $g=0$. Another useful NHPP model is the log linear model, which has the failure intensity function $\lambda(t)=e^{a+b t}$, where $a$ and $b$ are the parameters of the model. The log linear model can also be extended to accommodate interval or grouped failure data. Let 


\section{MODELING REPAIRABLE SYSTEM FAILURES}

$$
\begin{aligned}
v_{i} & =e^{\left(a+b t_{i}\right)}-e^{\left(a+b t_{i-1}\right)}, \\
w_{i} & =t_{i} e^{\left(a+b t_{i}\right)}-t_{i-1} e^{\left(a+b t_{i-1}\right)} \\
z_{i} & =t_{i}^{2} e^{\left(a+b t_{i}\right)}-t_{i-1}^{2} e^{\left(a+b t_{i-1}\right)} .
\end{aligned}
$$

The log-likelihood function for a series of $n$ time intervals is:

$$
L(a, b)=\sum_{i=1}^{n} d_{i} \ln \left(\frac{v_{i}}{b}\right)-\frac{v_{i}}{b}-\ln \left(d_{i} !\right)
$$

The first and second derivatives of the loglikelihood function are:

$$
\begin{aligned}
\frac{\partial L(a, b)}{\partial a} & =\sum_{i=1}^{n} \frac{d_{i} b-v_{i}}{b} \\
\frac{\partial L(a, b)}{\partial b} & =\sum_{i=1}^{n} \frac{\left(w_{i} b-v_{i}\right)\left(d_{i} b-v_{i}\right)}{b^{2} v_{i}}, \\
\frac{\partial^{2} L(a, b)}{\partial a^{2}} & =\sum_{i=1}^{n}-\frac{v_{i}}{b}, \\
\frac{\partial^{2} L(a, b)}{\partial a \partial b} & =\sum_{i=1}^{n}-\frac{\left(w_{i} b-v_{i}\right)}{b^{2}}, \\
, & \\
\frac{\partial^{2} L(a, b)}{\partial b^{2}} & =\sum_{i=1}^{n} \frac{d_{i} b^{3} v_{i} z_{i}+d_{i} b v_{i}^{2}-d_{i} b^{3} w_{i}^{2}-z_{i} b^{2} v_{i}^{2}}{b^{3} v_{i}^{2}} \\
& +\frac{2 w_{i} b v_{i}^{2}-2 v_{i}^{3}}{b^{3} v_{i}^{2}} .
\end{aligned}
$$

\section{Application with Real Data}

The real data used in this study consists of 25 time intervals to ball bearing failures in a conveyer belt in an automobile production. The failure occurrences are in intervals because the conveyer is only checked by the inspection team at certain times, referred to as inspection times (hours). There can be more than 1 failure in a certain time interval for which repair action is carried out. The time dependent covariate used is the number of maintenance actions taken throughout the study period.

Graphical methods are often used in modeling repairable systems to check trends in the data which then enables a reasonable model selection. Figure 1 displays the plot of the cumulative number of failures, $N\left(t_{i}\right)$ versus operating hours, $t_{i}$. Because data are failures within intervals, the graph was drawn using the upper interval point. The plot suggests that the use of a NHPP model might be appropriate because the failure rate appears to be inconsistent.

Figure 1: Cumulative Number of Failures vs. Time

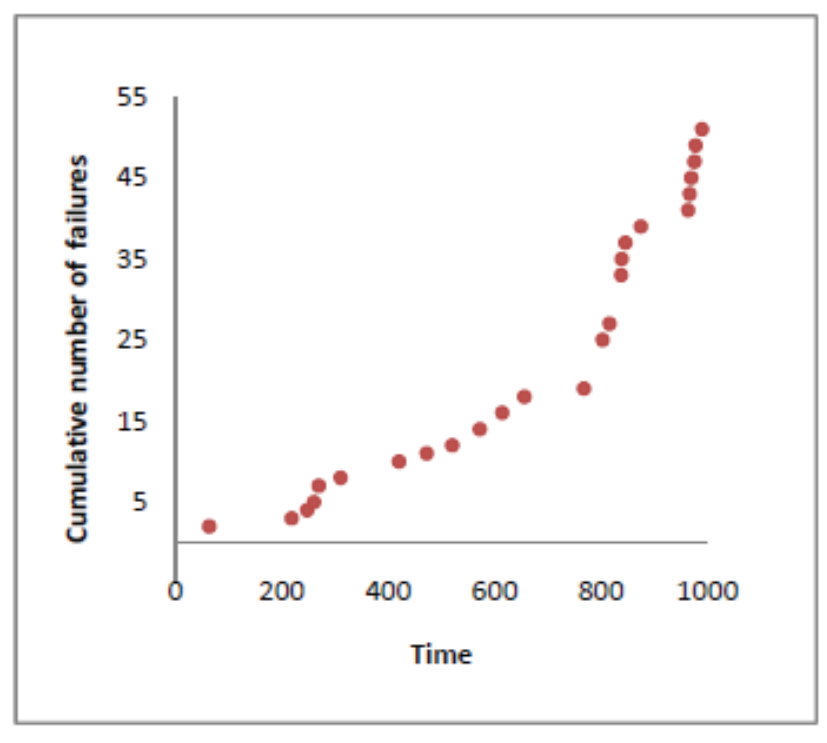

Table 1 shows the value of the parameter estimates and their standard errors when the data is fitted to the extended power law, power law, log-linear and HPP models. The table also shows the log likelihood value for each model at the estimated parameters. In the case of the extended power law model, the parameter estimate $g$ has a positive value; this implies that the maintenance action could not prevent the system from deteriorating with time. In addition, the estimate of $b$ shows a reliability improvement, but overall this fails to improve the system. All of the models show evidence of increasing failure intensity over time.

The extended power law model gives the highest log likelihood value, this implies that it fits the real data better than the other models. Figure 2 shows the estimates of the expected number of failures using the extended power law, power law, log linear and HPP models. The extended power law model shows the best fit for the real data, although the log linear appears to be a reasonable fit as well. The plot also shows 


\section{ARASAN \& EHSANI}

an obvious change in the slope towards the end on the process and certain data tend to form clusters, requiring further investigation.

Table 1: Parameter Estimates for Various Models

\begin{tabular}{cc}
\hline Ext. powerlaw & Powerlaw \\
\hline \hline$\hat{a}: 0.05542(0.11061)$ & $\hat{a}: 0.00007(0.14160)$ \\
$\hat{b}: 0.79426(0.31160)$ & $\hat{b}: 1.95071(0.28005)$ \\
$\hat{g}: 0.13092(0.03699)$ & $* * *$ \\
$L(\hat{a}, \hat{b}, \hat{g})=-61.89$ & $L(\hat{a}, \hat{b})=-66.60$ \\
\hline
\end{tabular}

\begin{tabular}{cc}
\hline Log linear & HPP \\
\hline \hline$\hat{a}:-4.60097(0.42546)$ & $\hat{a}: 0.05152(0.00721)$ \\
$\hat{b}: 0.00272(0.00058)$ & $* * *$ \\
$* * *$ & $* * *$ \\
$L(\hat{a}, \hat{b})=-62.33$ & $L(\hat{a})=-75.40$ \\
\hline
\end{tabular}

Figure 2: Real vs. Fitted for Several Models

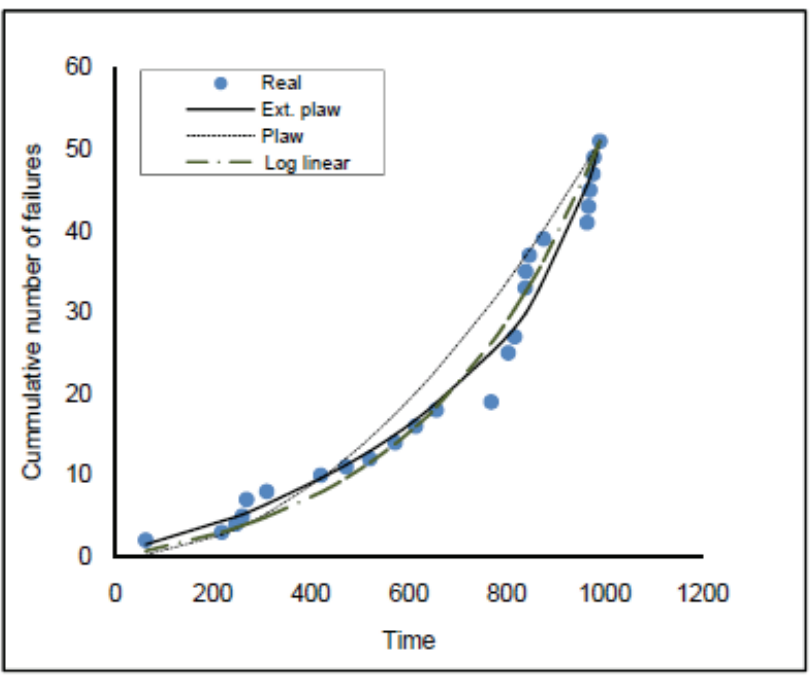

Hypothesis Testing and Confidence Intervals

If parameters $g$ and $b$ are significant then there is evidence of both maintenance effect and time trend within the model. The significance of the parameters $b$ and $g$ can be tested using likelihood ratio (LR) test. The idea of a LR test is to compare the maximized likelihood of two nested models, the full model and the reduced model. The reduced model is restricted by certain conditions in $H_{0}$.

Let $\hat{\theta}_{r}$ be the maximum likelihood estimator of the restricted model under $H_{0}$ and $\widehat{\theta}_{f}$ the maximum likelihood estimator of the full model. The maximized likelihood of the reduced model, $l\left(\widehat{\theta}_{r}\right)$ can never exceed the maximized likelihood of the full model, $l\left(\widehat{\theta}_{f}\right)$, because it is a subset of the full model. Thus, the ratio of the maximized likelihood of the reduced model to the full model is bounded between 0 and 1. A ratio close to 1 indicates that the reduced model is close to the full model whereas a ratio close to 0 indicates that the two models are very different and the reduced model is unacceptable. The likelihood ratio statistic for testing $H_{0}$ versus $H_{l}$ is the given by:

$$
\Psi=-2\left[L\left(\widehat{\theta}_{r}\right)-L\left(\widehat{\theta}_{f}\right)\right] .
$$

For a large sample size, $\Psi$ is approximately $\chi_{(\nu)}^{2}$, where $\nu$ is the number of parameters in the full model minus the number of parameters in the reduced model. The test statistic for testing the significance of the parameter, $g$, is 9.41 , which is higher than $\chi^{2}(0.05,1)=3.841$, thus implying that the effect of $g$ is significant at the 0.05 level. The test statistic for testing the significance of parameter $b$, is 27.014 , thus implying that the effect of $b$ is also significant at the 0.05 level. Thus, it may be concluded that the extended power law model is the most suitable model for the data.

Confidence intervals for the expected number of failures over interval $(a, b)$, $E[N(a, b)]=\mu(a, b)$ can be obtained by using the log normal distribution. The variance of an estimator can be calculated using the Delta method. The Delta method uses the $2^{\text {nd }}$ order Taylor expansion to approximate the variance of a function of random variables. Thus, 


\section{MODELING REPAIRABLE SYSTEM FAILURES}

$$
\begin{aligned}
& \operatorname{Var}(\hat{\mu}(a, b))=\left(\frac{\partial \mu(a, b)}{\partial a}\right)^{2} \operatorname{Var}(\hat{a})+ \\
& \left(\frac{\partial \mu(a, b)}{\partial b}\right)^{2} \operatorname{Var}(\hat{b})+\left(\frac{\partial \mu(a, b)}{\partial g}\right)^{2} \operatorname{Var}(\hat{g}) \\
& +2\left(\frac{\partial \mu(a, b)}{\partial a}\right)\left(\frac{\partial \mu(a, b)}{\partial b}\right) \operatorname{Cov}(\hat{a}, \hat{b}) \\
& +2\left(\frac{\partial \mu(a, b)}{\partial a}\right)\left(\frac{\partial \mu(a, b)}{\partial g}\right) \operatorname{Cov}(\hat{a}, \hat{g}) \\
& +2\left(\frac{\partial \mu(a, b)}{\partial b}\right)\left(\frac{\partial \mu(a, b)}{\partial g}\right) \operatorname{Cov}(\hat{b}, \hat{g}) .
\end{aligned}
$$

Following this, the confidence interval for $\mu(a, b)$ is

$$
\left[\hat{\mu}(a, b) e^{-\frac{Z_{\frac{\alpha}{2}} \sqrt{\operatorname{Var}(\hat{\mu}(a, b))}}{\hat{\mu}(a, b)}}, \hat{\mu}(a, b) e^{\frac{Z_{\frac{\alpha}{2}} \sqrt{\operatorname{Var}(\hat{\mu}(a, b))}}{\hat{\mu}(a, b)}}\right] .
$$

Figure 3: Confidence Interval for $\mu(a, b)$

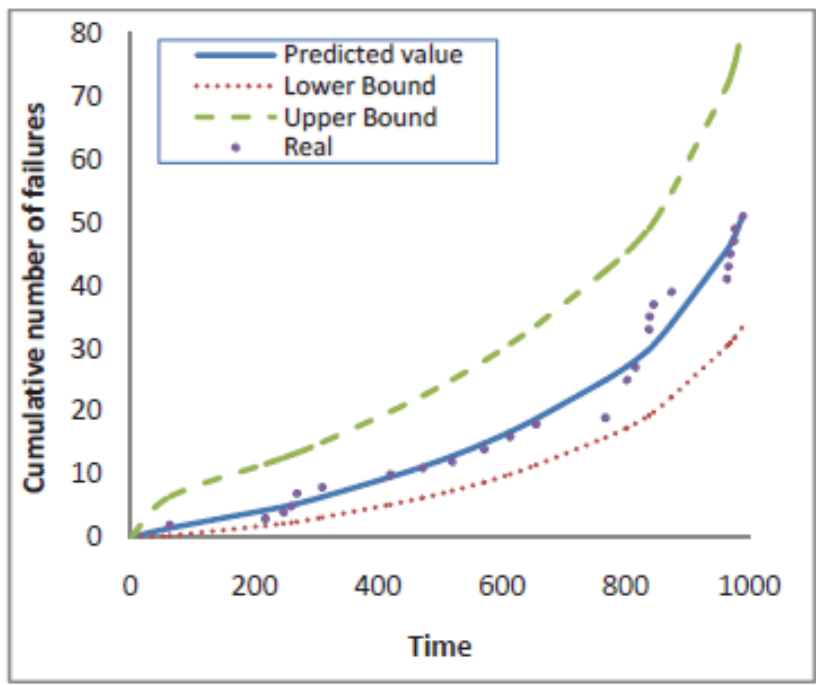

Another way to obtain the variance of $\hat{\mu}(a, b)$ is to use the bootstrap technique. Recently, alternative techniques requiring only minimal assumption have become popular. The bootstrapping technique was proposed by Efron (1993) and the procedure depends on how the bootstrap sampling is done. Efron (1993) showed that, in certain cases, the bootstrap estimate of variance or standard error can be used as an alternative for numerically estimating the traditional variance or standard error estimate.

Several different methods for generating bootstrap samples exist, namely parametric and nonparametric sampling procedures. This study utilizes the parametric bootstrap sampling procedure where B bootstrap samples of size $n$ are generated from an assumed parametric distribution. The number of failures over interval $(a, b)$ follows a Poisson distribution with mean $\mu(a, b)$. Thus, random samples can be generated from the Poisson distribution and bootstrap estimates of the mean, $\widehat{\theta}^{b}$, can be calculated where $b=1,2, \cdots, B$ are estimates calculated from each of the bootstrap samples of size $n$.

The bootstrap estimate of the variance of $\mu(a, b)$ is

$$
\widehat{\operatorname{Var}}_{B}=\frac{1}{B-1} \sum_{b=1}^{B}\left(\widehat{\theta}^{b}-\widehat{\theta}_{(.)}\right)^{2},
$$

where

$$
\widehat{\theta}_{(.)}=\sum_{b=1}^{B} \frac{\widehat{\theta}^{b}}{B}
$$

Following this, the confidence interval for $\mu(a, b)$ can be obtained in the similar way as

$$
\left[\hat{\mu}(a, b) e^{-\frac{Z_{\frac{\alpha}{2}} \sqrt{\widehat{\hat{V a r}} B}}{\hat{\mu}(a, b)}}, \hat{\mu}(a, b) e^{\frac{Z_{\frac{\alpha}{2}} \sqrt{\hat{\operatorname{Var}} B}}{\hat{\mu}(a, b)}}\right] .
$$

Figure 4 shows the $95 \%$ confidence interval for the expected cumulative number of failures using the bootstrap standard error estimate. This shows that the interval estimation using the bootstrap standard error estimate provides a good alternative and is slightly narrower than the traditional method.

\section{Conclusion}

This article proposed the use of the extended power law model for repairable systems with interval or grouped failure data and a time dependent covariate. The model reduces to the power law and HPP as a special case, thus it is convenient and useful. The model also allows incorporation and analysis of both time trend 


\section{ARASAN \& EHSANI}

Figure 4: Confidence Interval for $\mu(a, b)$ Using Bootstrap Standard Error

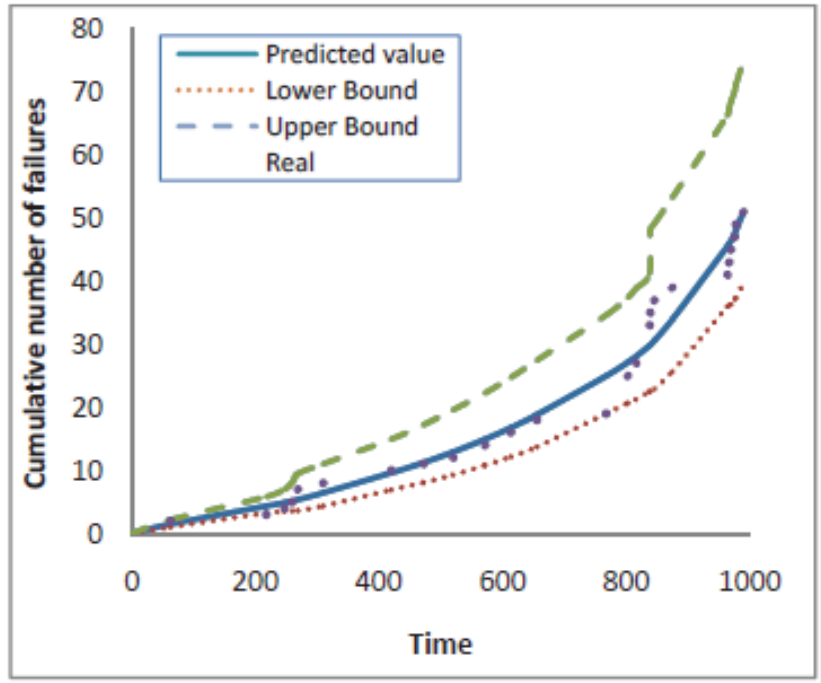

and covariate effects simultaneously. More research may be done by implementing the methods discussed herein to other repairable system models to determine if similar results are obtained. The use of other types of log-linear and linear models that can incorporate interval failure data with covariates should also be investigated.

The parametric bootstrap computer based technique was also employed to obtain the variance estimate for the estimated expected number of failures Alternative computer intensive techniques are simpler to implement and - in many cases - provide better estimates than traditional methods. Bootstrapping techniques are useful particularly when traditional methods become unreliable and certain assumptions are not satisfied. The high capability of modern day computers makes these methods practical.

Other parametric bootstrapping techniques and block jackknifing techniques for confidence interval estimation could also be explored. There may also be chances of applying other bootstrap confidence interval estimates such as percentile bootstrap, bootstrap-t and $\mathrm{BCa}$. These intervals are usually known to be more reliable and give better coverage probabilities and, as noted by Arasan (2008), are more symmetrical. However, their use with repairable system data should be done with caution; some modifications are also likely necessary to avoid violating the basic assumptions.

\section{References}

Arasan, \& Lunn. (2008). Alternative interval estimation for parameters of bivariate exponential model with time varying covariate. Computational Statistics, 23, 605-622.

Atwood, C. L. (1992). Parametric estimation of time-dependent failure rates for probabilistic risk assessment. Reliability Engineering and System Safety, 37, 181-194.

Brown, C. (1975). On the use of indicator variable for studying the time dependence of parameters in a response-time model. Biometrics, 31, 863-872.

Cook, R. J., \& Lawless, J. F. (2007). The statistical analysis of recurrent events. New York: Springer.

Cox, D. R., \& Lewis, P. A. (1966). Statistical analysis of series of events. London: Methuen.

Crow, L. H. (1974). Reliability analysis for complex, repairable systems. In Reliability and biometry, F. Proschan \& R. J. Serfling, Eds, 379-410. Philadelphia, PA: SIAM.

Duanne, J. T. (1964). Learning curve approach to reliability monitoring. IEEE Transactions on Aerospace, 2, 563-566.

Efron, B., \& Tibshirani, R. J. (1993). An introduction to the bootstrap. New York, NY: Chapman and Hall.

Gasmi, S., Love, C. E., \& Kahle, W. A. (2003). General repair, proportional hazards, framework to model complex repairable systems. IEEE Transactions on Reliability, 52(1), 26-32.

Guo, H. R., Liao, H., Zhao, W., \& Mettas, A. (2007). A new stochastic model for systems under general repairs. IEEE Transactions on Reliability, 56(1), 40-49.

Guo, H. R., Zhao, W., \& Mettas, A. (2006). Practical methods for modeling repairable systems with time trends and repair effects. Annual Reliability and Maintainability Symposium, 182-188. 


\section{MODELING REPAIRABLE SYSTEM FAILURES}

Jeng, S. L., \& Meeker, W. Q. (2000) Comparison for approximate confidence interval procedure for type I censored data. Technometrics, 42(2), 135-148.

Kaminskiy, M., \& Krivtsov, V. (1998). A Monte Carlo approach to repairable system repairable analysis, probabilistic safety assessment and management. New York, NY: Springer.

Kijima, M. (1989). Some results for repairable systems with general repair. Journal of Applied Probability, 26, 89-102.

Kijima, M., \& Sumita, N. (1986). A useful generalization of renewal theory: Counting process governed by non-negative Markovian increments. Journal of Applied Probability, 23, 71-88.

Lawless, J. F., \& Thiagarajah, K. (1996). A point-process model incorporating renewals and time trends, with application to repairable systems. Technometrics, 38(2), 131138.

Lawless, J. F., \& Zhan, M. (1998). Analysis of interval-grouped recurrent-event data using piecewise constant rate functions. The Canadian Journal of Statistics, 26, 549-565.

Meeker, W. Q., \& Escobar, L. A. (1998). Statistical methods for reliability data. New York, NY: Wiley.
Park, S., Jun, H., Kim, B. J., \& Im, G. C. (2008). Modeling of water main failure rates using the log-linear rocof and the power law process. Water Resource Management, 22, 1311-1324.

Samira, E. F. \& Arasan, J. (2009). A counting process for repairable system under general repair with repair history and time varying covariate. Proceedings of $17^{\text {th }}$ National Symposium on Mathematical Sciences, 2, 823828.

Vesely, W. E. (1977). Estimating common cause failure probabilities in reliability and risk analysis: Marshall-Olkin specialization. In Nuclear System Reliability Engineering and Risk Assessment, J. B. Fussell \& G. R. Burdick, Eds., 314-341. Philadelphia, PA: SIAM.

Wang, H., \& Pham, N. (1996). Optimal age dependent preventive maintanance policies with imperfect maintanance. International Journal of Reliability, Quality and Safety Engineering, 3, 119-135.

Yanez, M., Joglar, F., \& Modarres, M. (2002). Generalized renewal process for analysis of repairable systems with limited failure experience. Reliability Engineering and System Safety, 77, 167-180. 